\title{
Profils de multiples comportements à risque pour la santé des étudiants universitaires et leurs liens avec la santé mentale : utilisation de l'analyse des classes latentes
}

\author{
M. Y. Kwan, Ph. D. (1,2); K. P. Arbour-Nicitopoulos, Ph. D. (3); E. Duku, Ph. D. (4); G. Faulkner, Ph. D. (3,5)
}

Cet article a fait l'objet d'une évaluation par les pairs.

Diffuser cet article sur Twitter

\section{Résumé}

Introduction : Les campus universitaires et collégiaux sont sans doute les derniers milieux au sein desquels il est possible d'aborder de façon globale la question de la santé d'une grande proportion de la population de jeunes adultes. Il est important que les promoteurs de la santé saisissent en quoi consistent les difficultés collectives auxquelles font face les étudiants et qu'ils comprennent mieux les modèles plus larges de comportements liés au mode de vie qui se manifestent au cours de cette période de la vie. L'objectif de notre étude a été de déterminer des catégories de comportements à risque pour la santé modifiables et d'étudier la relation entre ces catégories et divers paramètres relevant de la santé mentale au sein d'un vaste échantillon d'étudiants universitaires canadiens.

Méthodologie : Des étudiants de premier cycle $(n=837$, âge moyen $=21$ ans $)$ de l'Université de Toronto ont répondu à l'enquête National College Health Assessment (NCHA) (évaluation nationale de la santé dans les collèges) qui comprend environ 300 éléments, dont des évaluations de l'état de santé, de la santé mentale et des comportements à risque pour la santé des étudiants. Nous avons réalisé une analyse des classes latentes pour relever des profils en fonction de huit comportements à risque pour la santé connus (consommation de marijuana, consommation d'autres drogues illégales, rapports sexuels à risque, tabagisme, excès occasionnel d'alcool, mauvaise alimentation, inactivité physique, manque de sommeil).

Résultats : Nous avons obtenu un modèle à trois catégories axé sur les profils de comportement des étudiants : étudiants «typiques ", " à risque élevé » et " relativement en bonne santé ». Nos résultats ont par ailleurs montré que les étudiants à risque élevé ont déclaré souffrir d'un niveau de stress considérablement plus élevé que celui des étudiants typiques $\left(\chi^{2}[1671]=7,26 ; p<0,01\right)$.

Conclusion : Les étudiants les plus susceptibles d'adopter de multiples comportements à risque pour la santé ont fait état d'une moins bonne santé mentale, particulièrement en ce qui concerne le stress. Bien que l'on doive interpréter ces conclusions avec prudence en raison du taux de réponse de $28 \%$, celles-ci suggèrent néanmoins que l'on pourrait envisager des interventions ciblées auprès de groupes d'étudiants ayant des profils similaires de multiples comportements à risque pour la santé.

Mots-clés : étudiants universitaires, santé mentale, comportements à risque pour la santé, analyse des classes latentes
Points saillants

- Cette étude analyse les comportements à risque pour la santé modifiables et leurs effets sur la santé chez des étudiants canadiens de premier cycle universitaire.

- On peut distinguer trois profils liés aux comportements à risque pour la santé chez ces étudiants.

- Nos résultats confortent l'idée que, au sein de la population des étudiants à l'université, adopter de multiples comportements à risque pour la santé est en lien avec une santé mentale médiocre.

- Des interventions ciblant divers groupes présentant des comportements à risque pour la santé similaires pourraient être nécessaires et une plus grande importance devrait être attachée à la promotion des comportements favorables à la protection de la santé sur les plans de l'alimentation, de l'activité physique et du sommeil.

\section{Introduction}

Les comportements adoptés ou maintenus par les étudiants au collège ou à l'université sont susceptibles de modeler leur futur état de santé et celui des générations à venir, en raison du rôle qu'ils sont amenés à jouer plus tard à titre de parents et de leaders. Au vu des quelque 20 millions d'étudiants inscrits dans plus

\section{Rattachement des auteurs :}

1. Département de médecine familiale, Université McMaster, Hamilton (Ontario), Canada

2. École de médecine Michael-G.-DeGroote, campus régional de Niagara, Université McMaster, St. Catharines (Ontario), Canada

3. Faculté de kinésiologie et d'éducation physique, Université de Toronto, Toronto (Ontario), Canada

4. Département de psychiatrie et de neurosciences du comportement et Offord Centre for Child Studies, Université McMaster, Hamilton (Ontario), Canada

5. School of Kinesiology, University of British Columbia, Vancouver (Colombie-Britannique), Canada

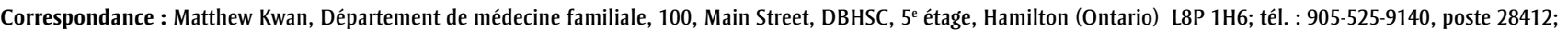
téléc. : 905-527-4440; courriel : kwanmy@mcmaster.ca 
de 6000 établissements postsecondaires aux États-Unis et au Canada ${ }^{1}$, cette influence sur la santé de la population pourrait se révéler considérable.

L'Okanagan Charter for Health Promoting Universities and Colleges de 2015 (charte Okanagan des universités et des collèges faisant la promotion de la santé) est une charte internationale élaborée en vue de promouvoir la santé auprès des étudiants universitaires et collégiaux. Elle affirme que les établissements postsecondaires doivent se concentrer sur la promotion de la santé et que le campus constitue un milieu idéal pour la mise en œuvre d'initiatives de promotion de la santé ${ }^{2}$. Comme les campus sont des installations subventionnées disposant souvent de programmes et de personnel soutenant les interventions et les efforts de promotion de la santé, les étudiants postsecondaires pourraient bénéficier d'une diffusion solide de messages sur la santé.

L'obtention du diplôme d'études secondaires est souvent considérée comme la première grande transition de la vie, qui commande des ajustements considérables dans diverses sphères ${ }^{3}$. La promotion de la santé au cours de cette période est essentielle, car une forte proportion de la population commence alors à adopter des comportements à risque pour la santé 4 . Le début de l'âge adulte est marqué par une augmentation considérable de la consommation de tabac, de marijuana et d'alcool et par une diminution des comportements favorables à la protection de la santé comme l'activité physique et une saine alimentation ${ }^{4.5}$. Étant donnée la plus grande autonomie dont bénéficient les jeunes adultes, on peut s'attendre à ce qu'ils modifient leurs comportements : leurs inhibitions précédentes à l'égard des comportements à risque pourraient s'atténuer en raison d'une supervision adulte moins importante et d'une perception accrue que bon nombre des comportements à risque pour la santé constituent des comportements adultes normaux ${ }^{6}$. Les établissements postsecondaires, qui accueillent un nombre considérable de jeunes adultes au sein d'un espace commun, pourraient constituer le dernier milieu où soit analysable de façon globale la santé d'une population relativement autonome.

Quoiqu'il soit vraisemblable que de nombreux comportements à risque pour la santé soient interreliés, la majorité des études épidémiologiques sur les comportements liés à la santé menées auprès d'étudiants postsecondaires ne tiennent pas compte de ces interrelations potentielles $^{7}$ : elles portent généralement sur plusieurs comportements à risque pour la santé en tant que mesures dépendantes distinctes $^{8}$. Il est pourtant important que les promoteurs de la santé saisissent en quoi consistent les difficultés collectives auxquelles font face les étudiants. De plus, les chercheurs ont à saisir quels modèles plus larges de comportements liés au mode de vie se manifestent au cours de cette période de la vie.

De nouvelles techniques statistiques, dont l'analyse des classes latentes (ACL), permettent d'examiner conjointement plusieurs comportements à risque pour la santé ou plusieurs variables dépendantes. Plus précisément, les ACL permettent de relever un nombre réduit de catégories de personnes dont les réponses sont similaires $^{9}$. La classification de la population en groupes sur la base de profils de comportements communs pourrait améliorer la portée, l'utilisation et l'efficacité des interventions ciblant concomitamment plusieurs comportements à risque pour la santé modifiables ${ }^{10}$.

Laska et ses collègues ${ }^{11}$ ont été les premiers à publier une étude s'appuyant sur une ACL pour modéliser des profils de comportements à risque pour la santé modifiables associés au mode de vie d'étudiants postsecondaires. Ils ont relevé quatre profils de comportements pouvant servir à adapter les activités de promotion de la santé au sein des campus en ciblant ces groupes spécifiques d'étudiants. Il existe toutefois peu de données sur la façon dont cette typologie des comportements (profils communs de comportements à risque pour la santé) pourrait être reliée à des paramètres relevant de la santé mentale comme le stress, l'anxiété et la dépression.
Le bien-être psychologique des étudiants postsecondaires est de plus en plus reconnu comme étant important ${ }^{2.7}$. Il semble pourtant que les jeunes adultes d'aujourd'hui souffrent davantage de stress et de symptômes de dépresssion ${ }^{12}$, et qu'ils présentent une plus grande détresse psychologique que l'ensemble de la population ${ }^{13}$. L'examen de la relation entre multiples comportements à risque pour la santé et paramètres relevant de la santé mentale pourrait permettre d'établir les profils comportementaux des étudiants postsecondaires et de déterminer comment ces profils sont liés à la santé mentale.

Le principal objectif de cette étude a consisté à utiliser une ACL pour déterminer les profils de comportements à risque pour la santé d'un échantillon d'étudiants universitaires canadiens. Dans la lignée des travaux de Laska et de ses collègues ${ }^{11}$, nous avons également étudié le lien entre les catégories latentes relevées et certains paramètres relevant de la santé mentale, à savoir la fatigue, le stress, la dépression, l'anxiété et la détresse psychologique.

\section{Méthodologie}

\section{Base de données et échantillon}

Nous avons recueilli des données au printemps 2009 en utilisant l'enquête $\mathrm{NCHA}^{14}$. (Pour de plus amples renseignements sur ce sujet, consulter le site Web de l'American College Health Association [ACHA $]^{15}$ ). L'enquête NCHA, dont la fiabilité et la validité ont fait l'objet d'une évaluation approfondie auprès d'étudiants collégiaux et universitaires des États-Unis ${ }^{15}$, comporte environ 300 questions, dont des évaluations de l'état de santé, de la santé mentale et des comportements liés à la santé des étudiants.

Pour obtenir notre échantillon d'étude, nous avons sélectionné aléatoirement 5000 des 50000 étudiants du campus St. George de l'Université de Toronto pour participer à l'enquête. Nous avons envoyé à chacun, par courriel, une invitation à participer à l'enquête en ligne à partir d'un site Web sécurisé géré par l'ACHA. 
Trois courriels de rappel les invitant à répondre à l'enquête en ligne ont été ensuite envoyés dans le mois.

Notre échantillon était constitué de 837 étudiants à temps plein de premier cycle (taux de réponse à l'enquête de $27,9 \%$ ). L’âge moyen de l'échantillon était de 20,92 ans (écart-type : 3,73) et les participants étaient en majorité des femmes $(64 \%)$ et de race blanche $(65 \%)$. Les caractéristiques de notre échantillon sont présentées dans le tableau 1.

Le conseil d'éthique de la recherche de l'Université du Toronto a approuvé nos protocoles d'étude.

\section{Mesures}

Les participants ont fourni des renseignements sur leur âge, leur sexe, leur taille et leur poids et leur lieu de résidence (sur le campus, hors campus ou au domicile des parents) ainsi que sur leur statut d'étudiant (temps plein ou temps partiel, année d'étude).

Les huit comportements à risque pour la santé que nous avons mesurés étaient le manque d'activité physique, une consommation insuffisante de fruits et de légumes, le manque de sommeil, le tabagisme, la consommation de marijuana, la consommation de drogues illégales, l'excès occasionnel d'alcool et un comportement sexuel à risque. Les cinq paramètres relevant de santé mentale mesurés étaient la fatigue, le stress, une dépression diagnostiquée, une anxiété diagnostiquée et de la détresse psychologique.

\section{Tabagisme (cigarettes), marijuana et drogues illégales}

Comme dans les enquêtes du Système canadien de surveillance ${ }^{16}$, nous avons questionnéles participants sur leur consommation de tabac, de marijuana et de drogues illégales : " Au cours des 30 derniers jours, pendant combien de jours avezvous consommé les substances suivantes...? » Les choix de réponse allaient de « jamais » à " pas dans les 30 derniers jours » et à « tous les jours dans les 30 derniers jours ». Nous avons créé deux

TABLEAU 1

Caractéristiques des participants et des effets sur la santé mentale

\begin{tabular}{|c|c|c|c|c|}
\hline & $\begin{array}{c}\text { Total } \\
(N=837)\end{array}$ & $\begin{array}{l}\text { Hommes } \\
(n=299)\end{array}$ & $\begin{array}{l}\text { Fem } \\
(\mathbf{n}=\end{array}$ & $\begin{array}{l}\text { Imes } \\
538)\end{array}$ \\
\hline Âge, moyenne (ET) & $21,00 \quad(4,25)$ & $21,43(5,76)$ & 20,75 & $(3,10)$ \\
\hline \multicolumn{5}{|l|}{ Lieu de résidence, n (\%) } \\
\hline Sur le campus & $183(21,9)$ & $71(23,7)$ & 112 & $(20,8)$ \\
\hline Hors campus & $654(78,0)$ & $228(76,3)$ & 426 & $(79,2)$ \\
\hline \multicolumn{5}{|l|}{ Année d'étude, n (\%) } \\
\hline Première & $199(23,8)$ & $69(23,1)$ & 130 & $(24,2)$ \\
\hline Deuxième & $228(27,2)$ & $85(28,4)$ & 143 & $(26,6)$ \\
\hline Troisième & $196(23,4)$ & $72(24,1)$ & 124 & $(23,0)$ \\
\hline Quatrième & $162(19,4)$ & $54(18,1)$ & 108 & $(20,1)$ \\
\hline Cinquième ou supérieure & $52(6,2)$ & $19(6,4)$ & 33 & $(6,1)$ \\
\hline \multicolumn{5}{|l|}{ Stress, $\mathbf{n}(\%)$} \\
\hline Non stressé & $362(43,2)$ & $139(46,5)$ & 223 & $(41,4)$ \\
\hline Stressé & $473(56,5)$ & $158(52,8)$ & 315 & $(58,6)$ \\
\hline Aucune réponse & $2(0,2)$ & $2(0,7)$ & & \\
\hline \multicolumn{5}{|l|}{ Fatigue, n (\%) } \\
\hline Non fatigué & $453(54,1)$ & $160(53,5)$ & 293 & $(54,5)$ \\
\hline Fatigué & $380 \quad(45,4)$ & $138(46,2)$ & 242 & $(45,0)$ \\
\hline Aucune réponse & $4(0,5)$ & $1(0,3)$ & 3 & $(0,6)$ \\
\hline \multicolumn{5}{|l|}{ Anxiété diagnostiquée, n (\%) } \\
\hline Non & $766(91,5)$ & $281(94,0)$ & 485 & $(90,1)$ \\
\hline Oui & $71(8,5)$ & $18(6,0)$ & 53 & $(9,9)$ \\
\hline \multicolumn{5}{|c|}{ Dépression diagnostiquée et suivi d'un traitement, n (\%) } \\
\hline Non & $775(92,6)$ & $283(94,6)$ & 492 & $(91,4)$ \\
\hline Oui & $62(7,4)$ & $16(5,4)$ & 46 & $(8,6)$ \\
\hline Détresse psychologique, moyenne (ET) & $3,55(2,64)$ & $3,04(2,60)$ & 3,90 & $(0,24)$ \\
\hline
\end{tabular}

Abréviation : ET, écart-type.

Remarque : Les variables du lieu de résidence, de l'année d'étude, du stress, de la fatigue, de l'anxiété diagnostiquée et de la dépression diagnostiquée sont indiquées en effectifs de participants et en pourcentages alors que l'âge et la détresse psychologique sont des moyennes (accompagnées des écarts-types [ET]).

catégories dichotomiques, soit les consommateurs (au cours des 30 derniers jours) et les non-consommateurs (aucune consommation au cours des 30 derniers jours).

\section{Abus occasionnel d'alcool}

Nous avons demandé aux participants : « Au cours des 15 derniers jours, combien de fois avez-vous consommé 5 boissons alcoolisés ou plus en une seule occasion? » À l'instar d'études précédentes ${ }^{17}$, nous avons considéré comme buveurs excessifs les participants ayant répondu avoir consommé de l'alcool de façon excessive une fois ou plus au cours des 15 derniers jours et comme buveurs non excessifs les participants n'ayant pas consommé d'alcool de façon excessive au cours des 15 derniers jours.

\section{Comportement sexuel à risque}

Nous avons demandé aux participants : " $\mathrm{Au}$ cours des 12 derniers mois, avezvous en raison de votre consommation d'alcool [...] eu des rapports sexuels non protégés? » Les réponses étaient soit oui, soit non (que ce soit car les participants n'avaient pas bu ou car ils n'avaient pas adopté ce comportements à risque pour la santé). À l'instar d'études antérieures sur le sujet ${ }^{11}$, nous avons considéré une réponse positive comme témoignant d'un comportement sexuel à risque. 


\section{Manque d'activité physique}

Nous avons estimé les comportements portant sur une activité physique modérée ou vigoureuse (APMV) à l'aide des deux questions suivantes : "Pendant combien de jours au cours des 7 derniers jours avez-vous : fait de l'exercice cardio ou aérobique d'intensité modérée (qui provoque une augmentation notable de la fréquence cardiaque, comme la marche rythmée) pendant au moins 30 minutes? » et " avez-vous fait de l'exercice cardio ou aérobique d'intensité élevée (qui provoque une augmentation importante de la fréquence respiratoire ou cardiaque) pendant au moins 20 minutes? » Les choix de réponse des participants allaient de 0 à 7 jours. Conformément aux Directives canadiennes en matière d'activité physique $^{18}$, nous avons additionné les résultats des deux questions et avons classé les participants dans les catégories "insuffisamment actifs » (étudiants qui font de l'APMV moins de 3 jours par semaine) ou "suffisamment actifs » (étudiants qui font de l'APMV 4 jours ou plus par semaine).

\section{Consommation insuffisante de fruits et de légumes}

Nous avons demandé aux participants "Combien de portions de fruits et de légumes consommez-vous généralement par jour? (1 portion correspondant à 1 fruit de taille moyenne, à $1 / 2$ tasse de fruits ou légumes frais, congelés ou en conserve, à $3 / 4$ de tasse de jus de fruits ou de légumes, à 1 tasse de verdure, à $1 / 4$ de tasse de fruits séchés.) " Les choix de réponse étaient aucune portion par jour, 1 à 2 portions par jour, 3 à 4 portions par jour et 5 portions ou plus par jour. Nous avons créé deux catégories de réponses visant à témoigner d'une consommation suffisante ou non de fruits et de légumes ( 5 portions ou plus de fruits et de légumes par jour ou moins de 5 portions de fruits et de légumes par jour). La consommation de 5 portions ou plus par jour est inférieure aux recommandations canadiennes ${ }^{19}$, mais cette mesure s'harmonise avec les anciennes recommandations des États-Unis ${ }^{20}$.

\section{Manque de sommeil}

Nous avons demandé aux participants "Pendant combien de jours au cours des 7 derniers jours avez-vous suffisamment dormi pour vous sentir reposé au réveil le matin? " Comme dans le cadre d'études antérieures ${ }^{11}$, nous avons séparé les réponses en deux catégories reflétant un sommeil suffisant (reposant 4 nuits par semaine ou plus) ou non (moins de 4 nuits) sur la majorité des nuits de la semaine.

\section{Fatigue}

Nous avons demandé aux participants « Au cours des 7 derniers jours, combien de fois vous êtes-vous senti fatigué, exténué ou endormi pendant le jour? » Nous avons considéré que les participants souffraient d'une fatigue plus élevée que la normale s'ils avaient répondu 4 jours ou plus, et qu'ils éprouvaient une fatigue normale s'ils avaient répondu moins de 4 jours.

\section{Stress}

Une seule question a été posée sur le sujet : " Comment qualifieriez-vous le niveau de stress que vous avez ressenti au cours des 12 derniers mois? » Nous avons considéré que les participants ayant répondu " aucun stress ", " moins élevé que le niveau moyen de stress » ou « niveau moyen de stress » éprouvaient un niveau normal de stress, et que les participants ayant répondu "plus élevé que le niveau moyen de stress » ou "stress intense " souffraient d'un stress de niveau plus élevé que le stress d'intensité normale.

\section{Diagnostic de dépression ou d'anxiété}

Nous avons demandé aux participants "Au cours des 12 derniers mois, avezvous reçu un diagnostic ou un traitement de la part d'un professionnel pour l'un des problèmes suivants? » Nous avons séparé les réponses pour la dépression et l'anxiété en " oui » (oui, diagnostiquée mais non traité; oui, traitée à l'aide de médicaments; oui, traitée au moyen d'une psychothérapie; oui, traitée au moyen de médicaments et d'une psychothérapie; oui, autre traitement) ou " non » (non diagnostiquée ou traitée).

\section{Détresse psychologique}

Nous nous sommes appuyés sur six questions pour évaluer la détresse psychologique en demandant aux participants : "Avez-vous déjà ressenti que votre situation était sans espoir; que vous étiez dépassé par tout ce que vous deviez accomplir; que vous étiez exténué (pour d'autres raisons que l'activité physique); que vous étiez très seul; que vous étiez très triste; que vous souffriez d'une anxiété insurmontable? » Les résultats des réponses positives (oui au cours des 12 derniers mois) ont été cumulés et combinés pour rendre compte de la détresse psychologique.

\section{Analyse statistique}

Nous avons réalisé une ACL pour obtenir les profils (ou catégories) sous-jacents de comportements à risque pour la santé en fonction des réponses des étudiants aux questions sur l'excès occasionnel d'alcool, la consommation de tabac, de marijuana et de drogues illégales, les comportements sexuels à risque, le manque d'activité physique, la consommation insuffisante de fruits et de légumes et le manque de sommeil. Afin de nous assurer que la solution du maximum de vraisemblance était bien choisie dans ces modèles, nous avons effectué 200 itérations de chaque modèle (de deux à quatre). Nous avons également généré de façon aléatoire des valeurs de départ afin d'obtenir véritablement le maximum de vraisemblance. Nous avons déterminé, grâce à la version 6.1 du logiciel Mplus $^{21}$, le nombre optimal de catégories latentes selon l'ajustement statistique du modèle en utilisant le critère d'information d'Akaike (CIA), le critère d'information bayésien (CIB), le critère d'information bayésien corrigé en fonction de la taille de l'échantillon (CIBA), l'entropie ainsi que les tests du rapport de vraisemblance de Lo-Mendell-Rubin (LMR) et de Lo-Mendell-Rubin (BLMR) avec méthode bootstrap ${ }^{22}$.

Pour choisir le nombre approprié de catégories latentes, nous avons analysé un modèle à deux catégories et avons comparé ses résultats avec des modèles successifs intégrant un nombre accru de catégories latentes (jusqu'à quatre). Nous avons examiné des estimations spécifiques pour les modèles à trois et à quatre catégories afin de choisir une spécification définitive en fonction de l'interprétation possible des résultats ${ }^{11}$. Un test d'invariance des mesures a révélé 
qu'aucune différence importante n'était décelable entre les sexes entre les modèles avec et sans contrainte. Nous avons intégré aux modèles définitifs les covariables suivantes : sexe, année et lieu de résidence (sur le campus ou non). Nous avons utilisé la fonction auxiliaire du logiciel Mplus afin d'examiner la relation entre l'appartenance à une catégorie et les paramètres relevant de la santé mentale que sont le stress, la fatigue, l'anxiété, la dépression et la détresse psychologique dans un modèle de régression logistique multinomiale. Nous avons considéré chaque paramètre relevant de la santé mentale comme une variable dépendante distincte et nous avons présenté les résultats du test d'égalité des moyennes de toutes les catégories obtenus à l'aide d'imputations multiples fondées sur les probabilités a posteriori.

\section{Résultats}

\section{Conclusions de l'analyse des classes latentes}

Un modèle à trois catégories d'étudiants de premier cycle a permis l'ajustement optimal du modèle (voir le tableau 2) compte tenu des huit comportements à risque pour la santé, soit le manque d'activité physique, la consommation insuffisante de fruits et de légumes, le manque de sommeil, le tabagisme, l'excès occasionnel d'alcool, la consommation de marijuana, la consommation de drogues illégales et un comportement sexuel à risque, tous ayant été corrigés en fonction du sexe, de l'année d'étude et du lieu de résidence (sur le campus ou non). Les probabilités de réponse pour chaque catégorie - la probabilité d'adopter chacun des comportements à risque pour la santé sont présentées sur la figure 1 et décrites ci-dessous.

\section{Catégorie 1 : étudiants typiques}

Ce groupe se caractérise par le rassemblement le plus fréquent de comportements à risque pour la santé modifiables et représente environ les deux tiers $(65,7 \%)$ de l'échantillon. Ces étudiants étaient peu susceptibles de fumer, d'avoir des comportements sexuels à risque ou de consommer de la marijuana ou des drogues illégales (probabilités entre 1,6\% et 6,4\%), mais ils étaient également peu susceptibles d'adopter des comportements favorables à la protection de la santé. Les probabilités étaient faibles qu'ils mangent des fruits et des légumes en quantité suffisante $(6,2 \%)$, qu'ils soient actifs physiquement $(12,2 \%)$ et qu'ils dorment suffisamment $(27,6 \%)$ (figure 1). Notons que parmi les trois catégories relevées, les étudiants typiques étaient associés aux probabilités les plus faibles d'excès occasionnel d'alcool.
Catégorie 2 : étudiants à risque élevé

Ce groupe se caractérise par l'adoption de comportements néfastes pour la santé touchant les huit sphères. À l'instar du groupe des étudiants typiques, ces étudiants étaient peu susceptibles d'adopter des comportements favorables à la protection de la santé (c.-à-d. que la probabilité de manger des fruits et des légumes en quantité suffisante, d'être actifs physiquement et de dormir suffisamment était inférieure à $25 \%$ ). À la différence des premiers, ils étaient les plus susceptibles d'adopter des comportements à risque pour la santé. Les probabilités de fumer, de consommer de la marijuana et de faire un excès occasionnel d'alcool étaient les plus importantes, variant entre $55,4 \%$ et $61,8 \%$, et les probabilités de consommer des drogues illégales (35,7 \%) et d'avoir des rapports sexuels non protégés $(27,8 \%)$ étaient également les plus élevées. Cette catégorie représentait environ $20 \%$ de l'échantillon d'étudiants.

\section{Catégorie 3 : étudiants relativement en bonne santé}

Ce groupe, qui constituait $14,5 \%$ de l'échantillon, représentait les étudiants dont le mode de vie est le plus sain. De façon similaire au groupe des étudiants typiques, ils étaient peu susceptibles de fumer, d'avoir des rapports sexuels non protégés et de consommer de

TABLEAU 2

Critères d'évaluation de l'ajustement du modèle

\begin{tabular}{|c|c|c|c|c|c|c|}
\hline & \multicolumn{3}{|c|}{ Ajustement du modèle sans covariables } & \multicolumn{3}{|c|}{ Ajustement du modèle avec covariables } \\
\hline & 2 catégories & 3 catégories & 4 catégories & 2 catégories & 3 catégories & 4 catégories \\
\hline Logarithme du rapport de vraisemblance & $-2940,43$ & - 2913,89 & $-2903,32$ & $-2924,82$ & - 2897,72 & $-2880,59$ \\
\hline $\mathrm{CIA}$ & 5914,87 & 5879,78 & 5876,36 & 5889,63 & 5859,44 & 5849,17 \\
\hline $\mathrm{CIB}$ & 5995,27 & 6002,75 & 6042,17 & 5984,18 & 6010,71 & 6057,18 \\
\hline CIBA & 5941,29 & 5920,18 & 5931,36 & 5920,66 & 5909,09 & 5917,49 \\
\hline Entropie & 0,725 & 0,635 & 0,753 & 0,714 & 0,658 & 0,635 \\
\hline LMR & $227,80^{*}$ & $52,23^{*}$ & 20,80 & 234,22 & $53,53^{*}$ & 33,85 \\
\hline BLMR & - $3056,22^{*}$ & - $2940,43^{*}$ & - 2913,89 & - 3043,37 & - $2924,82^{*}$ & - 2897,72 \\
\hline Estimation des paramètres & 17 & 26 & 35 & 20 & 32 & 44 \\
\hline
\end{tabular}

Abréviations : BLMR, test du rapport de vraisemblance de Lo-Mendell-Rubin selon la méthode bootstrap; CIA, critère d'information d'Akaike; CIB, critère d'information bayésien; CIBA, Critère d'information bayésien corrigé en fonction de la taille de l'échantillon; LMR, test du rapport de vraisemblance de Lo-Mendell-Rubin.

${ }^{*} p<0,05$. 
FIGURE 1

Probabilités de réponse à chaque comportement à risque pour la santé en fonction des trois catégories (étudiants typiques, à risque élevé et relativement en bonne santé) issues de l'analyse des classes latentes des comportements des étudiants

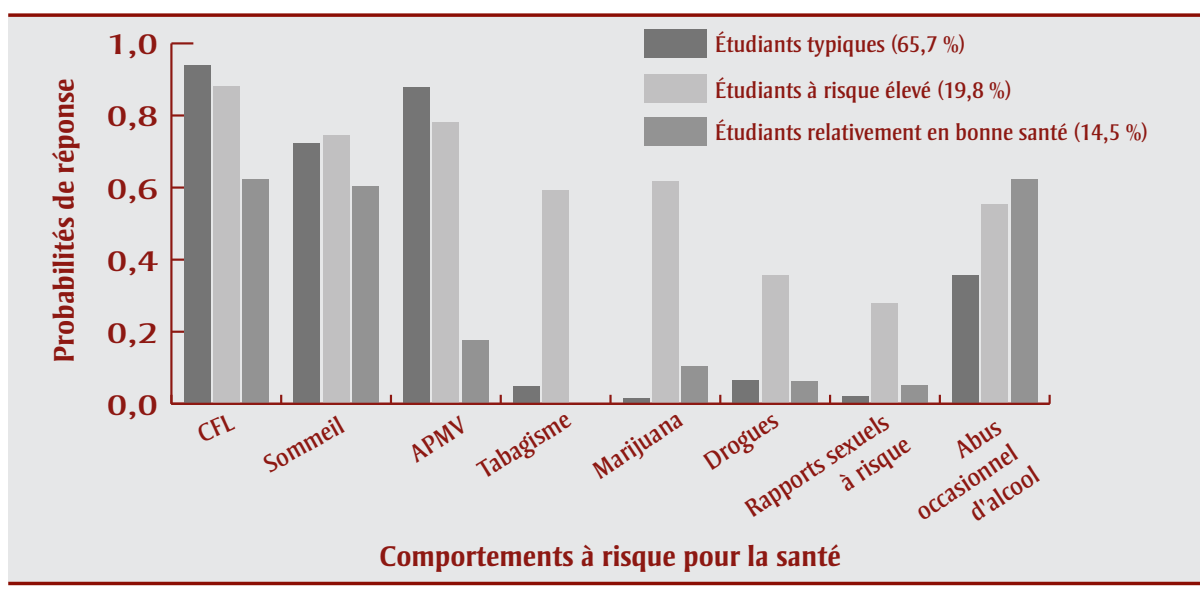

Abréviations : CFL, consommation de fruits et de légumes; APMV, activité physique modérée à vigoureuse.

Remarques : Nous avons inversé les codes de la CFL, du sommeil et de l'APMV afin que les plus faibles probabilités d'adopter ces comportements témoignent d'un risque élevé.

Les étudiants typiques représentent 65,7\% de l'échantillon, les étudiants à risque élevé, 19,8 \% et les étudiants relativement en bonne santé, $14,5 \%$.

la marijuana et des drogues illégales (probabilités allant de $0 \%$ pour le tabagisme à 6,3 \% pour la consommation de drogues illégales). La probabilité d'adopter des comportements favorables à la protection de la santé était au mieux faible, bien qu'ils étaient les plus susceptibles de consommer au moins 5 portions de fruits et de légumes par jour $(37,7 \%)$ et de dormir suffisamment $(39,8 \%)$. Les étudiants relativement en bonne santé, toutefois, étaient très susceptibles d'être actifs physiquement $(82,4 \%$ d'entre eux répondaient aux recommandations en matière d'APMV), mais également très susceptibles de faire des excès occasionnels d'alcool (62,3\%) (figure 1).

\section{Appartenance à une catégorie et paramètres relevant de la santé mentale}

Dans l'ensemble, les différences étaient statistiquement significatives entre les niveaux de stress rapportés en fonction de la catégorie $\left(\chi^{2}[2833]=8,55 ; p<0,05\right)$. Plus précisément, les étudiants du groupe à risque élevé ont affirmé souffrir de niveaux de stress considérablement plus élevés que les étudiants typiques $\left(\chi^{2}[1671]=7,26 ; p<0,01\right)$, mais pas plus élevés que les étudiants relativement en bonne santé $\left(\chi^{2}\right.$ [1285] = 3,12; $p=0,07)$. Bien qu'elles ne soient pas significatives, les statistiques descriptives indiquent que les étudiants à risque élevé présentent la plus grande prévalence d'autres problèmes de santé mentale, dont les niveaux les plus élevés de fatigue, d'anxiété diagnostiquée, de dépression diagnostiquée et de détresse psychologique déclarées par les participants. À l'opposé, les étudiants relativement en bonne santé présentaient la plus faible prévalence de la majorité des problèmes de santé mentale examinés. (Voir le tableau 3 pour obtenir des descriptions complètes des paramètres relevant de la santé mentale en fonction de l'appartenance à une catégorie.)

\section{Analyse}

Nos résultats ont mis en évidence différents profils importants de comportements à risque pour la santé au sein d'un échantillon d'étudiants canadiens de premier cycle universitaire. Nous avons défini trois groupes en fonction des profils communs de comportements à risque pour la santé. Ces groupes étaient constitués soit des étudiants susceptibles d'adopter plusieurs comportements à risque pour la santé, comme le tabagisme, un excès occasionnel d'alcool, la consommation de drogues (marijuana et autres drogues illégales) et des comportements sexuels à risque, soit des étudiants " typiques " ou " relativement en bonne santé » n'adoptant généralement pas ces comportements à risque. Il est troublant de constater que se dégage toutefois de notre analyse le fait que la grande majorité des étudiants, quelle que soit leur catégorie, était très peu susceptible d'adopter des comportements favorables à la protection de la santé. En particulier, tous les groupes présentaient des probabilités élevées similaires pour ce qui est du manque du sommeil et de la consommation insuffisante de fruits et de légumes.

La santé publique met généralement l'accent sur la réduction des comportements à risque pour la santé, en particulier ceux associés à des maladies non transmissibles, comme le tabagisme et la consommation d'alcool ${ }^{23}$. Nos conclusions montrent cependant que les comportements à risque pour la santé les plus répandus dans les campus sont les habitudes que les étudiants n'adoptent pas (celles relatives à l'activité physique, à la consommation de fruits et de légumes et au sommeil). Même les étudiants relativement en bonne santé étaient susceptibles de ne pas bénéficier de suffisamment de sommeil et de consommer moins que la moitié des 8 à 10 portions quotidiennes de fruits et de légumes ${ }^{19}$ recommandées à l'échelle nationale. Ces profils de comportements pourraient témoigner des difficultés (p. ex. le temps requis pour faire de l'exercice physique ou le coût et l'accessibilité des fruits et des légumes) auxquelles font face les étudiants au cours de cette importante étape de transition en ce qui concerne l'autogestion de comportements complexes, dont ceux liés à l'activité physique, à l'alimentation et au sommeil.

Ces profils de comportements font également l'objet de préoccupations compte tenu des données probantes qui indiquent que le tabagisme, l'excès occasionnel d'alcool et le manque d'activité physique s'aggravent au cours de l'adolescence et au début de l'âge adulte $e^{4.24}$. Si les jeunes adultes ont tendance à cesser l'excès occasionnel d'alcool et le tabagisme au milieu de la vingtaine, l'inactivité physique 
TABLEAU 3

Comparaison des paramètres relevant de la santé mentale en fonction de l'appartenance à une catégorie selon le test d'égalité des moyennes appliqué à toutes les catégories

\begin{tabular}{|c|c|c|c|c|}
\hline & \multicolumn{4}{|c|}{$\begin{array}{l}\text { Probabilité de problèmes de santé mentale } \\
\qquad \%(E T)\end{array}$} \\
\hline & $\begin{array}{c}\text { Total } \\
(N=837)\end{array}$ & $\begin{array}{l}\text { Étudiants typiques } \\
(65,7 \%)\end{array}$ & $\begin{array}{c}\text { Étudiants à } \\
\text { risque élevé } \\
(19,8 \%)\end{array}$ & $\begin{array}{c}\text { Étudiants relativement } \\
\text { en bonne santé } \\
(14,5 \%)\end{array}$ \\
\hline Stress & $56,5^{*}$ & $54,1(2,2)^{\mathrm{a}}$ & $66,9(4,0)$ & $54,5 \quad(5,3)^{* *}$ \\
\hline Fatigue & 45,4 & $45,9(2,3)$ & $49,9(4,4)$ & $39,0 \quad(5,5)^{* *}$ \\
\hline Dépression diağnostiquée & 7,4 & $6,7(1,1)$ & $9,9(2,5)$ & $6,5 \quad(2,7)$ \\
\hline Détresse psychologique & $\begin{array}{r}3,55 \\
( \pm 2,64)\end{array}$ & $3,52(0,12)$ & $3,90(0,24)$ & $3,26 \quad(0,28)^{* *}$ \\
\hline
\end{tabular}

Abréviation : ET, écart-type

Remarque : Les statistiques sur la prévalence sont représentées en pourcentages, exception faite de la détresse psychologique dont les scores varient entre 0 et 6 . Les tailles d'échantillon sont estimées en fonction de la probabilité d'appartenance à l'une des catégories latentes.

${ }^{a}$ Diffère considérablement de la catégorie à risque élevé.

${ }^{*} p<0,05$.

${ }^{* *} p<0,09$.

continue à augmenter ${ }^{4}$. Cet ensemble de données probantes met en évidence la nécessité de veiller à ce que les efforts de promotion de la santé portent sur les comportements favorables à la santé encore insuffisamment intégrés tout autant que sur les comportements à risque pour la santé les plus couramment ciblés comme le tabagisme ou la consommation de drogues. Les initiatives comme Healthy Campus 2020 Framework (cadre pour la santé dans les campus) ont joué un rôle important dans l'augmentation d'une promotion visant l'alimentation et l'activité physique auprès des jeunes adultes. Toutefois, pour atteindre ces objectifs, il faut mettre en place davantage d'initiatives de promotion d'une vie saine et active au sein des campus.

Fait intéressant, et exception faite de l'excès occasionnel d'alcool, nous avons relevé des différences entre les catégories pour ce qui est de la façon dont les étudiants adoptent les comportements usuels présentant un risque pour la santé. Alors que la fréquence globale de ces comportements à risque pour la santé est relativement faible, un groupe d'étudiants semble adopter plusieurs de ces comportements à risque. Ceci semble indiquer que les stratégies actuelles de promotion de la santé sont efficaces de manière générale pour la majorité de la population étudiante de premier cycle, mais qu'il pourrait être nécessaire de mener une intervention ciblée auprès du groupe à risque. Cette dernière pourrait prendre la forme de matériel de promotion de la santé ciblant ces comportements à risque pour la santé de façon globale plutôt que séparément. Cibler conjointement plusieurs comportements à risque pour la santé pourrait permettre aux individus de faire un transfert de connaissances et d'expériences d'un comportement à un autre si les sphères s'y rapportant sont similaires ${ }^{26}$. L'idée est de provoquer le changement de l'un de ces comportements, ce qui est susceptible d'avoir un effet domino similaire à celui rapporté dans des études concernant la diminution concomitante de la consommation de marijuana et d'alcool en cas d'abandon du tabac ${ }^{26}$.

\section{Multiples comportements à risque pour la santé et paramètres relevant de la santé mentale}

Notre étude s'est également penchée sur la façon dont ces profils de multiples comportements à risque pour la santé étaient liés à la santé mentale. Dans l'ensemble, nos conclusions corroborent le fait selon lequel l'adoption de plusieurs comportements à risque pour la santé est associée à une moins bonne santé mentale. Bien que les résultats n'aient pas été statistiquement significatifs, le niveau de fatigue, de stress et de détresse psychologique des étudiants relativement en bonne santé était moins élevé que celui des étudiants à risque élevé. Ces conclusions revêtent une grande importance, la détresse psychologique étant souvent considérée comme un indicateur fiable de la santé $^{27}$ et une bonne gestion du stress et de la fatigue étant essentielle à la réussite scolaire ${ }^{28}$. Les études à venir doivent se pencher sur ces liens au fil du temps, car le caractère transversal de notre étude ne nous a pas permis de déterminer la direction de ces associations. Toutefois, le stress qu'éprouvent les étudiants constituant un élément central de l'initiative Healthy Campus 2020 Framework $^{25}$, il faut mettre sur pied des interventions ciblant conjointement les comportements à risque et le stress. La promotion de l'activité physique comme moyen de composer avec le stress constitue un exemple d'approche en matière d'intervention qui vise à la fois la santé mentale et la santé physique. Les futures études axées sur les étudiants à risque revêtiront également une grande importance, car les profils de comportements à risque de ces derniers témoignent peut-être de stratégies d'adaptation inadéquates. Des interventions 
pourraient viser à remplacer les stratégies d'adaptation nuisibles, comme le tabagisme et la consommation d'alcool, par des stratégies d'adaptation saines, comme un sommeil régulier et de l'activité physique.

\section{Points forts et limites}

Bien que notre étude fasse appel à une technique statistique relativement neuve pour examiner les profils de multiples comportements à risque pour la santé au sein d'une cohorte d'étudiants universitaires, nous devons néanmoins faire état de plusieurs de ses limites. En premier lieu, il existe de toute évidence un biais dans les réponses, car seulement $28 \%$ des étudiants de premier cycle invités à participer à l'enquête ont répondu à cette dernière. Bien que ce faible taux de réponse soit comparable à celui d'autres études aux États-Unis ${ }^{30}$ et au Canada ${ }^{5.6}$ utilisant les données d'enquête NCHA, nos conclusions doivent malgré tout être interprétées avec prudence.

En deuxième lieu, ces données se rapportent à des étudiants provenant d'une seule université, située dans un grand centre urbain. Quoique notre échantillon ait été largement représentatif des étudiants de premier cycle de l'Université de Toronto âge moyen de 21,1 ans et $56 \%$ de femmes ${ }^{29}$, nous sommes limités dans la généralisation de ces conclusions aux étudiants d'autres établissements postsecondaires.

En troisième lieu, si les outils d'autodéclaration volontaire comme l'enquête NCHA sont essentiels à la collecte de données sur la santé, l'analyse de données secondaires a ses limites. Par exemple, nous étions limités par les mesures de l'enquête et nous ne disposions pas de mesures validées des paramètres relevant de la santé mentale comme l'anxiété et la dépression. De plus, les comportements à risque pour la santé étaient mesurés sur différentes périodes (les 7, 15 ou 30 derniers jours). Si les mesures basées sur des périodes plus longues avaient pour but de relever les comportements à risque pour la santé moins courants (comme la consommation de drogues illégales), l'idéal aurait néanmoins été d’obtenir une évaluation uniforme pour chacun des comportements.

Enfin, bien que nos analyses aient tenu compte du sexe, du statut d'étudiant et du lieu de résidence comme covariables, d'autres facteurs individuels pourraient s'avérer utiles pour caractériser les diverses catégories. Les études à venir pourraient dresser des profils plus descriptifs de chacune des catégories afin que l'adaptation des interventions soit plus facile. Il faudra également mener des études longitudinales portant sur les mêmes étudiants au fil du temps afin de déterminer la direction des relations entre les comportements à risque pour la santé et les paramètres connexes, notamment la santé mentale.

\section{Conclusion}

La transition vers le début de l'âge adulte constitue une cible importante des efforts de promotion de la santé, et les campus postsecondaires sont des milieux idéaux pour aborder de façon globale la question de la santé de nombreux jeunes adultes. En réponse à l'appel à l'action de l'Okanagan Charter ${ }^{2}$, nos conclusions confirment la nécessité de considérer quels comportements liés à la santé sont ciblés par les services de santé des campus et elles montrent qu'il faut accorder une plus grande importance aux comportements de protection de la santé liés à l'alimentation, à l'activité physique et au sommeil. Avant tout, les promoteurs de la santé devront tenir compte des difficultés collectives auxquelles font face les étudiants et de la complexité associée au changement d'un comportement. Ils devront également tenir compte également du fait que les changements de comportement liés au mode de vie devront probablement s'opérer en concomitance avec le développement de compétences sur la maîtrise de soi, et ce, afin que les étudiants gèrent au mieux leurs divers intérêts pendant leur séjour au collège ou à l'université. Au delà, nos conclusions laissent penser qu'il existe un lien entre les profils associés à ces multiples comportements liés à la santé et la santé mentale des étudiants postsecondaires. Il faudra toutefois d'autres études pour connaitre les impacts au fil du temps de ces multiples comportements associés à la santé.

\section{Remerciements}

Guy Faulkner est titulaire d'une chaire de recherche appliquée en santé publique qui est financée par l'Agence de la santé publique du Canada (ASPC) en partenariat avec les Instituts de recherche en santé du Canada (IRSC).

Nous souhaitons remercier la Canadian Organization of University and College Health (association canadienne pour la santé dans les universités et les collèges) pour son soutien dans le cadre de ce projet.

\section{Références}

1. Association of Universities and Colleges of Canada (AUCC). Trends in higher education. Volume 1-Enrolment. Ottawa (Ont.): AUCC; 2011. PDF téléchargeable à partir du lien : http://www.cais.ca/ uploaded/trends-2011-vol1-enrolment-e.pdf

2. The Okanagan Charter for Health Promoting University and Colleges Working draft: a shared vision for health promoting universities and colleges. Kelowna (BC): Okanagan Charter; 2015. PDF téléchargeable à partir du lieu : http://international healthycampuses2015.sites.olt.ubc.ca /files/2015/06/Charter-Draft-One-Final -June-19-2015.pdf

3. Gall TL, Evans DR, Bellerose S. Transition to first-year university: patterns of change in adjustment across life domains and time. J Soc Clin Psychol. 2000;19:544-567. doi: 10.1521/jscp.2000.19.4.544.

4. Kwan MY, Cairney J, Faulkner GE, Pullenayegum EE. Physical activity and other health-risk behaviours during the transition into early adulthood. Am J Prev Med. 2012;42:14-20. doi: 10.1016/j. amepre.2011.08.026

5. Kwan MY, Faulkner GE, ArbourNicitopoulos KP, Cairney J. Prevalence of health-risk behaviours among Canadian post-secondary students: descriptive results from the National College Health Assessment. BMC Pub Health. 2013;13:548. doi: 10.1186/1471-2458-13-548. 
6. Arbour-Nicitopoulos KP, Kwan MY, Taman S, Lowe D, Faulkner GE. Normative beliefs in health behavioural practices in a college population. J Am Coll Health. 2011;59:191-196.

7. Bray SR Kwan MYW. Physical activity is associated with better health and psychological well-being during transition to university. J Am Coll Health. 2006; 55: 78-82.

8. Jackson CA, Henderson M, Frank JW, Haw SJ. An overview of prevention of multiple risk behaviour in adolescence and young adulthood. J Pub Health. 2012; 34 Suppl 1:i31-40. doi: 10.1093/pubmed/fdr113.

9. Slater MD, Flora JA. Health lifestyles: audience segmentation analysis for public health interventions. Health Educ Behav. 1991;18:221-233.

10. Hagenaars JA, McCutcheons AL (dir.). Applied latent class analysis. Cambridge (UK): Cambridge University Press; 2002.

11. Laska MN, Pasch KE, Lust K, Story M, Ehlinger E. Latent class analysis of lifestyle characteristics and health risk behaviours among college youth. Prev Sci. 2009;10(4):376-386. doi: 10.1007/ s11121-009-0140-2.

12. Alexander SJ, Harrison AG. Cognitive responses to stress, depression, and anxiety and their relationship to ADHD symptoms in first year psychology students. J Atten Dis. 2013;17:29-37

13. Stallman HM. Psychological distress in university students: a comparison with general population data. Aust Psychol. 2010; 45: 249-257.

14. American College Health Association (ACHA). American College Health Association - National College Health Assessment II: Reference Group Executive Summary Fall 2009. Linthicum (MD): American College Health Association; 2009.

15. American College Health Association. The American College Health Association National College Health Assessment (ACHA-NCHA), Spring 2005 Reference Group Data (Abridged). J Am Coll Health. 2006;55:5-16.

16. DeRuiter WK, Cairney J, Leatherdale S, Faulkner G. The period prevalence of risk behaviour co-occurrence among Canadians. Prev Med. 2016;85:11-16. doi:10.1016/j.ypmed.2015.11.026.
17. Nelson MC, Lust K, Story M, Ehlinger E. Alcohol use, eating patterns and weight behaviours in a university population. Am J Health Beh. 2009;33:227-237.

18. Agence de la santé publique du Canada (ASPC). Lignes directrices en matière d'activité physique. Guide d'activité physique canadien [Internet]. Ottawa (Ont.) : ASPC; 2006 [consulté le 1 août 2012, Guide non disponible]. Information en ligne à la page : http://www.phac-aspc. gc.ca/hp-ps/hl-mvs/pa-ap/03paap-fra.php

19. Santé Canada. Bien manger avec le Guide alimentaire canadien. Ottawa (Ont.), Santé Canada; 2011. Consultable à partir de la page : http://www.hc-sc.gc.ca/fn-an/food -guide-aliment/gen_prin-fra.php

20. US Department of Health and Human Services. Dietary guidelines for Americans, 8th ed. Washington (DC): US Department of Health and Human Services; 2005. Joint publication of the US Department of Agriculture.

21. Muthén LK, Muthén BO. Latent variable hybrids: overview of old and new models. Dans Hancock GR, Samuelsen KM (dir.), Advances in latent variable mixture models, p. 1-24. Charlotte (NC): Information Age Publishing, Inc.; 2010.

22. Das P, Horton R. Rethinking our approach to physical activity. Lancet. 2012;380(9838):189-90. doi: 10.1016/ S0140-6736(12)61024-1.

23. Wing Kwan MY, Bray SR, Martin Ginis KA. Predicting physical activity during transition to first-year university: an application of the Theory of Planned Behaviour. J Am Coll Health. 2009;58:45-52. doi: 10.3200/JACH.58.1.45-55.

24. Healthy People 2020 [Internet]. Washington (DC): US Department of Health and Human Services; [consulté en mai 2012]. http://www.healthypeople.gov

25. Barnett SM, Ceci SJ. When and where do we apply what we learn? A taxonomy for far transfer. Psychol Bull. 2002;128:612-637.

26. Adlaf EM, Demers A, Gliksman L (dir.). Enquête sur les campus canadiens 2004. Toronto (Ont.) : Centre de toxicomanie et de santé mentale; 2005.
27. Steptoe A, Tsuda A, Tanaka Y, Wardle J. Depressive symptoms, socio-economic background, sense of control, and cultural factors in university students from 23 countries. Int $\mathrm{J}$ Behav Med. 2007;14:97-102.

28. Nagane M. Relationship of subjective chronic fatigue to academic performance. Psychol Rep. 2004;95:48-52.

29. Keith DR, Hart CL, McNeil MP, Silver R, Goodwin RD. Frequent marijuana use, binge drinking and mental health problems among undergraduates. Am J Addict. 2015;24(6):499-506. doi: 10.1111/ajad.12201.

30. Paks-Aubé C, Liang X. University of Toronto Facts and Figures 2006. Toronto (Ont.) : University of Toronto, Office of the Vice-Provost, Planning and Budget; 2006. 